\title{
Image Learning of Electric Characteristics of Resistance, Capacitance, Inductance, and their Circuits by Java Programming
}

\author{
Masami Morooka ${ }^{1}$, Suhua Qian ${ }^{1}$, Midori Morooka ${ }^{2}$ \\ ${ }^{1}$ Department of Electrical Engineering, Fukuoka Inst. Tech, higashi-ku, Fukuoka 811-0295, Japan; \\ ${ }^{2}$ Flash Design Center, Micron Japan, Kamata 5-37-1, Ota-ku, Tokyo144-8721, Japan; \\ morooka@ee.fit.ac.jp
}

\begin{abstract}
Java programs with a graphical user interface (GUI) environment have been developed for an image learning of electric characteristics of resistance $(R)$, capacitance $(C)$, inductance $(L)$, and their circuits. Text fields of selected parameters for the numerical calculation of the differential equations to describe the electric characteristics of the circuit are set on the display, such as the frequency of applied voltage and the values of R, C, L, time increments, and calculation times. The calculation used Runge-Kutta method is initiated by clicking the start button after inputting the desired values into the text fields. The calculated results are plotted immediately after the completion of the calculation as a figure on the display for the simulation of the electric characteristics of the circuit, such as changes in the voltages and currents with time. By changing the values in the text fields, the new results are represented immediately, and the new electric characteristics of the new circuit can be easily simulated. These Java programs are useful in education applications for rapidly and accurately image learning for the electric characteristics of the circuit. This program is also useful for learning the phenomena expressed by ordinary differential equations.
\end{abstract}

Keywords: Image learning, characteristics of electric circuit, resistance - capacitance Inductance, Java programming, voltage - current characteristics of RCL circuits.Introductin

\section{INTRODUCTION}

In many situations, it is difficult to obtain the variation of the current and voltage in a circuit composed of a resistance (R), a capacitance (C), and an inductance (L). It is even more difficult to visualize the variation in the characteristics of the circuits when they are limited by each component. The electric characteristics of the circuit can be expressed by ordinary differential equations, which can be solved numerically using the Runge-Kutta method. With the wide use 
Masami Morooka, Suhua Qian and Midori Morooka; Image Learning of Electric Characteristics of Resistance, Capacitance, Inductance, and their Circuits by Java Programming, Transactions on Machine Learning and Artificial Intelligence, Volume 2 No 3, June (2014); pp: 01-19

of Java programming in a GUI (graphical user interface) environment and the rapid development of personal computers, the rapidly and accurately image learning for charge motions in electric and magnetic fields [1] and that for complicated diffusion of Au into Si [2] have been developed by the Java programming. In this paper, the ordinary differential equations for RCL circuits are solved numerically, and the voltage-current characteristics of the circuits are easily and accurately simulated using Java in a GUI environment. These Java programs are useful in education applications for rapidly and accurately image learning of electric characteristics of R, C, L, and their circuits.

\section{NUMERICAL METHOD FOR ELECTRIC CIRCUITS}

\subsection{Basic Equations for Voltage-Current Characteristics of R, C, and $L$}

The voltage $v(t)$ - current $i(t)$ characteristics of $R, C$, and $L$ are given as

$$
\begin{gathered}
i_{R}(t)=\frac{v_{R}(t)}{R} \\
i_{C}(t)=\frac{d q(t)}{d t}=C \frac{d v_{C}(t)}{d t} \\
v_{L}(t)=L \frac{d i_{L}(t)}{d t}
\end{gathered}
$$

where, $t$ is time and $q(t)$ is the charge of capacitance, and the subscripts refer to each of the components.

\subsection{Differential Equations and Numerical Method for Series RCL Circuits}

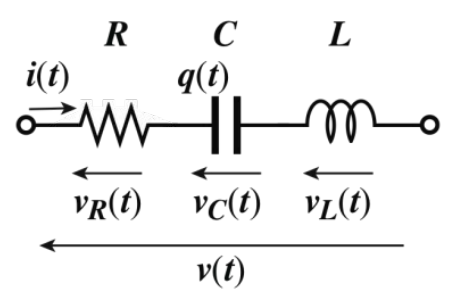

Figure 1: A series RCL circuit

The electric characteristics of a series circuit with an applied voltage $v(t)$ shown in Figure 1 are given by two ordinary differential equations:

$$
\begin{gathered}
\frac{d q(t)}{d t}=i(t), \\
\frac{d i(t)}{d t}=\frac{v(t)-R i(t)-q(t) / C}{L} .
\end{gathered}
$$

In this case, the increment functions for the forth-order Runge-Kutta method are 


$$
\begin{gathered}
k_{1}^{(1)}=i_{j}, \\
k_{2}^{(1)}=\frac{v(t)-R i_{j}-q_{j} / C}{L}, \\
k_{1}^{(2)}=i_{j}+k_{2}^{(1)} h / 2, \\
k_{2}^{(2)}=\frac{v(t+h / 2)-R\left(i_{j}+k_{2}^{(1)} h / 2\right)-\left(q_{j}+k_{1}^{(1)} h / 2\right) / C}{L}, \\
k_{2}^{(3)}=\frac{v(t+h / 2)-R\left(i_{j}+k_{2}^{(2)} h / 2\right)-\left(q_{j}+k_{1}^{(2)} h / 2\right) / C}{L}, \\
k_{1}^{(4)}=i_{j}+k_{2}^{(3)} h, \\
k_{2}^{(4)}=\frac{v(t+h)-R\left(i_{j}+k_{2}^{(3)} h\right)-\left(q_{j}+k_{1}^{(3)} h\right) / C}{L},
\end{gathered}
$$

where, $h$ is the increment of $t$ and the subscript ${ }_{j}$ represents the known variables at a given $t$. The details on the calculation of the increment functions are described in the Reference [1]. The unknown variables $q_{j+1}$, and $i_{j+1}$ at $t+h$ are given as

$$
\begin{gathered}
q_{j+1}=q_{j}+\frac{1}{6}\left(k_{1}^{(1)}+2 k_{1}^{(2)}+2 k_{1}^{(3)}+k_{1}^{(4)}\right), \\
i_{j+1}=i_{j}+\frac{1}{6}\left(k_{2}^{(1)}+2 k_{2}^{(2)}+2 k_{2}^{(3)}+k_{2}^{(4)}\right) .
\end{gathered}
$$

If the variables at a certain $t$ are known, the numerical values at $t+h$ can be obtained from Equations (14) and (15), and then the values for $t+2 h$, and $t+3 h$ and so on, can be obtained by repeating the calculations. The voltage for each of the components can be obtained using the calculated $q$ and $i$ with

$$
\begin{gathered}
v_{R}(t)=R i(t), \\
v_{C}(t)=\frac{q(t)}{C}, \\
v_{L}(t)=v(t)-v_{R}(t)-v_{C}(t) .
\end{gathered}
$$




\subsection{Differential Equations and Numerical Method for Parallel RCL Circuits}

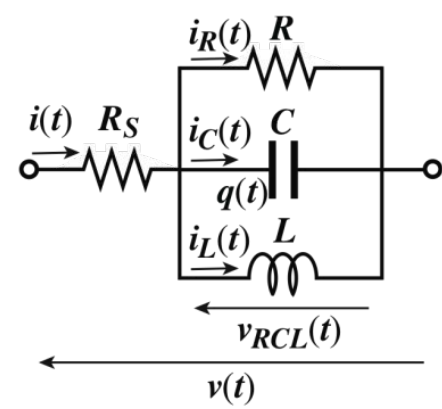

Figure 2: A parallel RCL circuit

The capacitance current depends on the change of the applied voltage, $d v(t) / d t=\Delta v / \Delta t$ as shown in Equation (2). If a voltage is applied directly to the capacitance, the capacitance current results in a very large numerical value depending on $h$. This occurs when the applied voltage is switched on or off, or a rectangle voltage is applied. Therefore, it is ideal to connect a series resistance that has a very small resistivity, in series with the capacitance for the numerical calculations, as shown $R_{S}$ in Figure 2. The electric characteristics of the parallel circuit shown in Figure 2 are given by two ordinary differential equations using $v_{R C L}(t)=q(t) / C$ :

$$
\begin{gathered}
\frac{d i_{L}(t)}{d t}=\frac{q(t)}{C L} \\
\frac{d q(t)}{d t}=\frac{v(t)}{R_{S}}-\frac{q(t)}{R_{S} C}-\frac{q(t)}{R C}-i_{L}(t) .
\end{gathered}
$$

In this case, the increment functions for the forth-order Runge-Kutta method are

$$
\begin{gathered}
k_{1}^{(1)}=\frac{q_{j}}{C L}, \\
k_{2}^{(1)}=\frac{v(t)}{R_{S}}-\frac{q_{j}}{R_{S} C}-\frac{q_{j}}{R C}-i_{L j}, \\
k_{1}^{(2)}=\frac{q_{j}+k_{2}^{(1)} h / 2}{C L}, \\
k_{2}^{(2)}=\frac{v(t+h / 2)}{R_{S}}-\frac{q_{j}+k_{2}^{(1)} h / 2}{R_{S} C}-\frac{q_{j}+k_{2}^{(1)} h / 2}{R C}-\left(i_{L j}+k_{1}^{(1)} h / 2\right), \\
k_{1}^{(3)}=\frac{q_{j}+k_{2}^{(2)} h / 2}{C L}, \\
k_{2}^{(3)}=\frac{v(t+h / 2)}{R_{S}}-\frac{q_{j}+k_{2}^{(2)} h / 2}{R_{S} C}-\frac{q_{j}+k_{2}^{(2)} h / 2}{R C}-\left(i_{L j}+k_{1}^{(2)} h / 2\right),
\end{gathered}
$$




$$
\begin{gathered}
k_{1}^{(4)}=\frac{q_{j}+k_{2}^{(3)} h}{C L}, \\
k_{2}^{(4)}=\frac{v(t+h)}{R_{S}}-\frac{q_{j}+k_{2}^{(3)} h}{R_{S} C}-\frac{q_{j}+k_{2}^{(3)} h}{R C}-\left(i_{L j}+k_{1}^{(3)} h\right) .
\end{gathered}
$$

The unknown variables $i_{L j+1}$ and $q_{j+1}$ at $t+h$ are given as

$$
\begin{gathered}
i_{L j+1}=i_{L j}+\frac{1}{6}\left(k_{1}^{(1)}+2 k_{1}^{(2)}+2 k_{1}^{(3)}+k_{1}^{(4)}\right), \\
q_{j+1}=q_{j}+\frac{1}{6}\left(k_{2}^{(1)}+2 k_{2}^{(2)}+2 k_{2}^{(3)}+k_{2}^{(4)}\right) .
\end{gathered}
$$

Other variables can be obtained using the calculated $i_{L}$ and $q$ :

$$
\begin{gathered}
v_{R C L}(t)=\frac{q(t)}{C}, \\
i(t)=\frac{v(t)-v_{R C L}(t)}{R_{S}}, \\
i_{R}(t)=\frac{v_{R C L}(t)}{R}, \\
i_{C}(t)=i(t)-i_{R}(t)-i_{L}(t) .
\end{gathered}
$$

\section{PROGRAMMING}

In a numerical calculation, values that alternate with time, such as the applied voltage, can be conveniently expressed as cosine functions instead of sine functions. For example, $v(t)=$ $v_{0} \cos (2 \pi f t)$. Here, $f$ is the frequency of the applied voltage. If we use $v(t)=v_{0} \sin (2 \pi f t)$, then $v=0$ at $f=0$ despite the existence of a direct electric voltage $v=v_{0}$. A rectangle voltage, which corresponds to switch on and off the DC voltage, can be expressed using the cosine function as shown below.

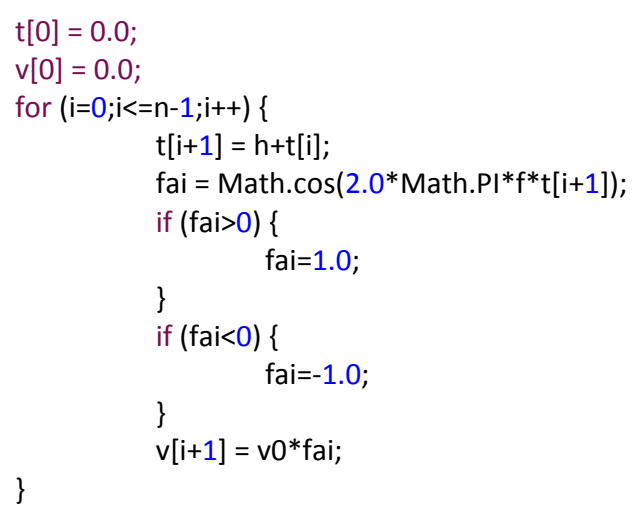

The main flow of the programming is shown below. 
Masami Morooka, Suhua Qian and Midori Morooka; Image Learning of Electric Characteristics of Resistance, Capacitance, Inductance, and their Circuits by Java Programming, Transactions on Machine Learning and Artificial Intelligence, Volume 2 No 3, June (2014); pp: 01-19

Step 1. Setting the text fields and buttons

$\downarrow$

Step 2. Calculating the variables using the Runge-Kutta method

$\downarrow$

Step 3. Selection of the minimum and maximum values of the calculated variables

$\downarrow$

Step 4. Re-calculation of the variable points on the display

$\downarrow$

Step 5. Insertion of the electric circuit

$\downarrow$

Step 6. Graphics

The basic programming for Steps 1 - 4 and Step 6 are same as the programming for the charge motion in electric and magnetic fields, and their details are previously reported [1]. In addition, it is useful to show the circuit as shown in Figures 1 and 2, while learning about the electric characteristics of RCL circuit (Step 5). A basic example of programming in Java to print $L$, $\mathrm{R}$ and $\mathrm{C}$ components and their values is shown below. Each component is prepared using only g.drawArc and g.drawLine statements. The functions of the main parts of the program are written as comments. The results from the program are shown in Figure 3.

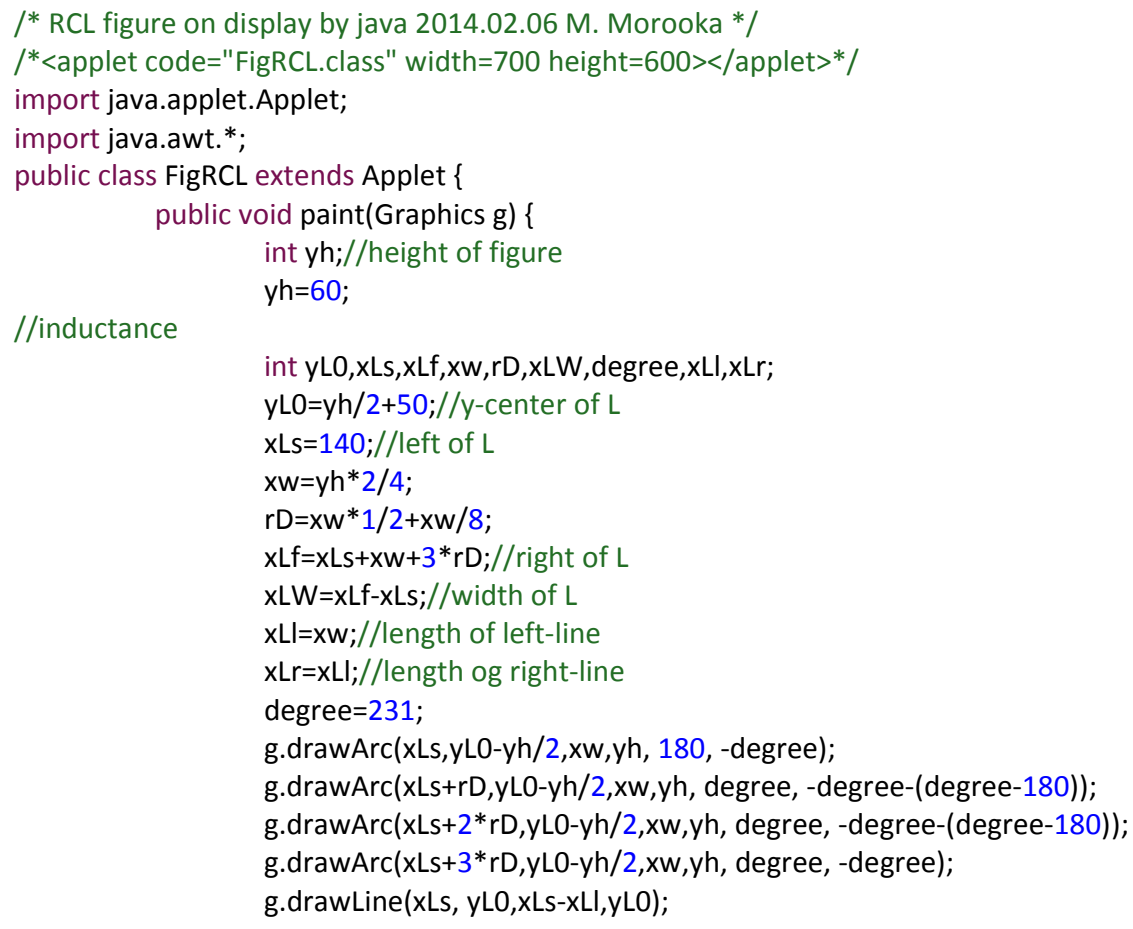


//resistance

g.drawLine(xLf, yLO,xLf+xLr,yLO);

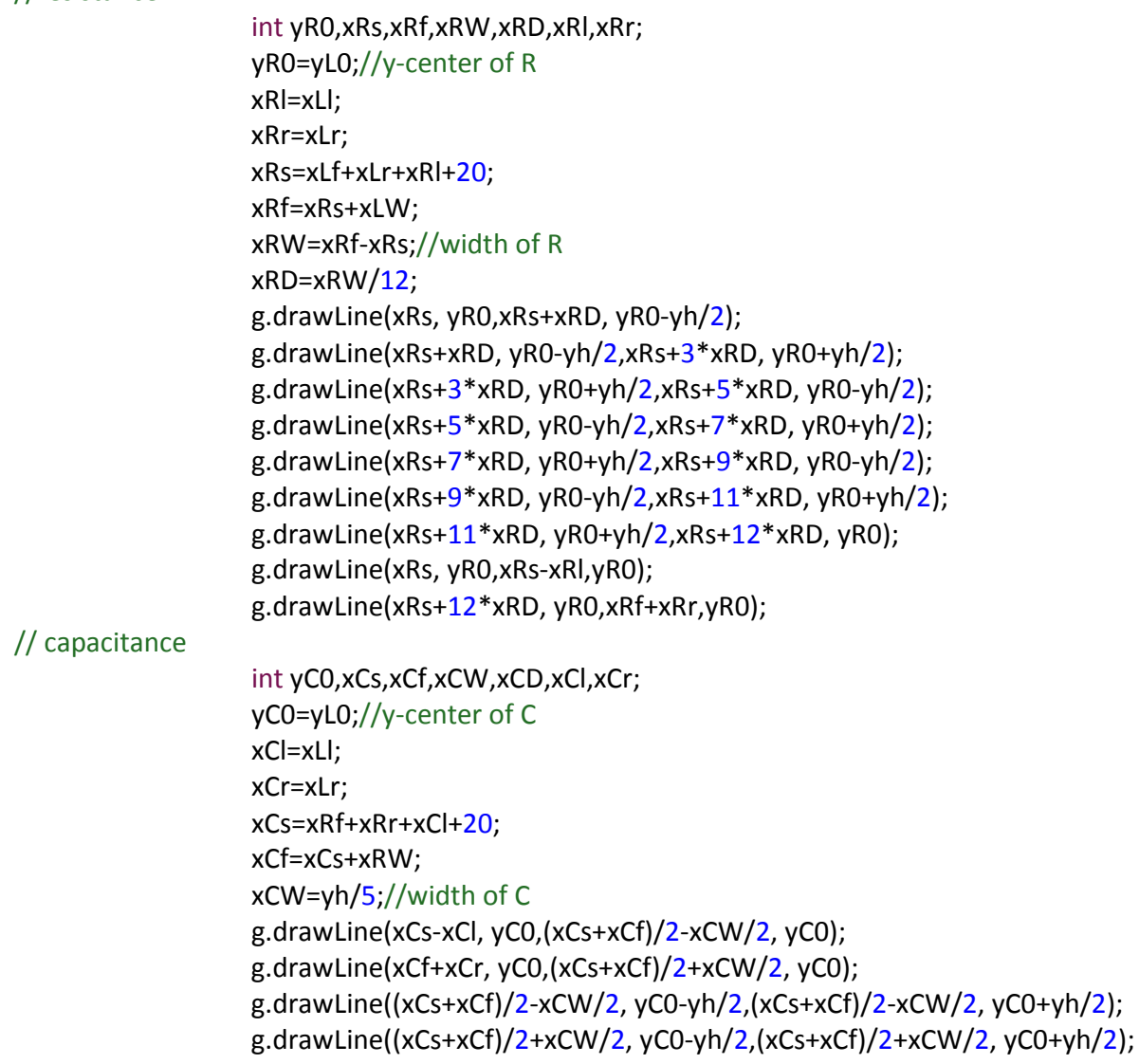

// print of values
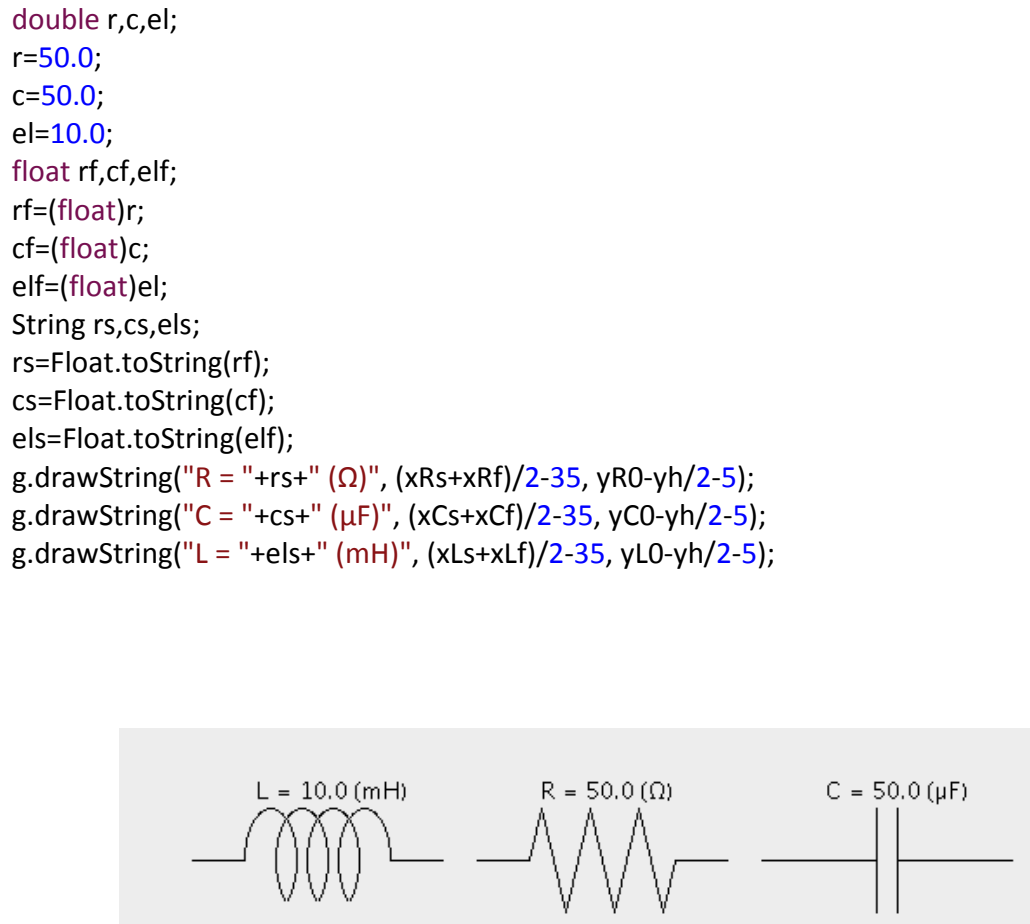

Figure 3: Basic print for the $L, R$, and $C$ and their values, displayed using the Java program 
Masami Morooka, Suhua Qian and Midori Morooka; Image Learning of Electric Characteristics of Resistance, Capacitance, Inductance, and their Circuits by Java Programming, Transactions on Machine Learning and Artificial Intelligence, Volume 2 No 3, June (2014); pp: 01-19

In this basic numerical calculation involving a series circuit, six text fields for the resistance coefficient $(R \Omega)$, the capacitance coefficient $(C \mu F)$, the inductance coefficient $(L m H)$, the frequency of the applied voltage $(f \mathrm{~Hz})$, the time increments ( $h \mathrm{sec})$, and the number of calculations $(n)$, are set on the display. For the calculations on parallel circuits, another text field for the series resistance $\left(R_{S} \Omega\right)$ is added. The inputted values for the $R, C$, and $L$ are automatically displayed on each of the components, as shown in Figure 3 . The calculated electric characteristics are plotted on the display, which is separated into two regions. The applied voltage $v(t)$, current $i(t)$, and charge $q(t)$ are plotted in the upper regions. Each of the voltages for the components, $v_{R}(t), v_{C}(t)$, and $v_{L}(t)$ in the series circuit, or each of the currents of the components, $i_{R}(t), i_{C}(t)$, and $i_{L}(t)$ in the parallel circuit are plotted in the lower regions.

\section{RESULTS}

The text fields are immediately presented on the display after the execution of the program by the appletviewer in Java. The calculation is initiated by clicking the start button after inputting values in the text fields. The figure of the circuit and the voltage-current characteristics are plotted immediately after the completion of the calculation. The time required to display the characteristics after clicking the start button is usually less than several seconds depending on the number of calculations and the performance of the computer. By changing the values in the text fields and re-clicking the start button, new characteristics can be obtained immediately.

\subsection{Electric Characteristics of Series RCL Circuit}

The typical AC characteristics of a series RCL circuit in which the resistance, capacitance, and inductance are comparably effective, that is, the impedance of each component is nearly equal, are shown in Figure 4. This figure shows the image on the display. The current is dominated by the resistivity, found by deducing the sum of the impedances of the capacitance and inductance, and the current results in same phase as the applied voltage. The phase of the charge is larger than that of the current by $\pi / 2$ as shown in Equation (2). The phase of the voltage across the resistance is same as that of the current. On the other hand, the phase of the capacitance voltage is larger than the current by $\pi / 2$ and that of the inductance voltage is less than the current by $\pi / 2$. The phase of the capacitance voltage is $\pi$ behind that of the inductance voltage. These basic electric characteristics of the $R, C$ and $L$ components are obtained from Equations (1) - (3), and can easily be shown visually on the display. By decreasing the value of $C$ in the text field by a factor of 10 , the new characteristics affected strongly by the capacitance, in which the phase of the current is less than that of the applied voltage by $\pi / 2$ and the larger part of the applied voltage is applied to the capacitance, are obtained immediately, as shown in Figure 5. At a small $t$ value, a transient characteristic appears, caused by the initial increase in the voltage from 0 to $v_{0}$. The increasing rate of the voltage is $v_{0} / h$ and depends on the inverse of the increment of time. The rate is usually large and results in a large transient effect on the characteristics of the capacitance, as shown in 
Figure 5. By increasing the value of $R$ in the text field by a factor of 10 , relative to that in Figure 4 , the new characteristics affected strongly by the resistance can be obtained immediately, and by increasing that of $L$ by a factor of 10 , the new characteristics affected strongly by the inductance can also be obtained immediately. The maximum value of each variable and the final times are also shown on the display. By changing the values in the text fields and reclicking the start button, the change of the electric characteristics in the series RCL circuit are easily and accurately recognized and are displayed immediately as an image on the display.

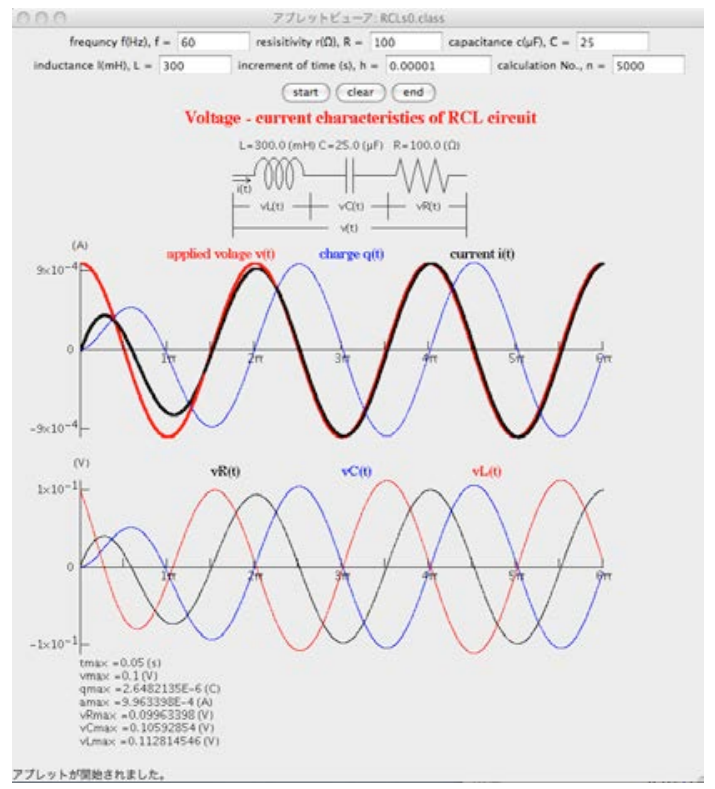

Figure 4: Typical AC characteristics of a series $\mathrm{RCL}$ circuit in which the $\mathrm{R}, \mathrm{C}$ and $\mathrm{L}$ components are comparably effective. The balues used are shown in the text fields, $f=60 \mathrm{~Hz}, R=100 \Omega, C=25 \mu \mathrm{F}$, and $\mathrm{L}=300 \mathrm{mH}$. $v(t)$, $i(t)$, and $q(t)$ are shown in the upper regions. $v_{R}(t), v_{c}(t)$, and $v_{L}(t)$ are shown in the lower regions. The current results in similar phase to the applied voltage, and the phase of the voltage for each component is different by $\pi / 2$ each other. The maximum values of the variables are also shown on the display 


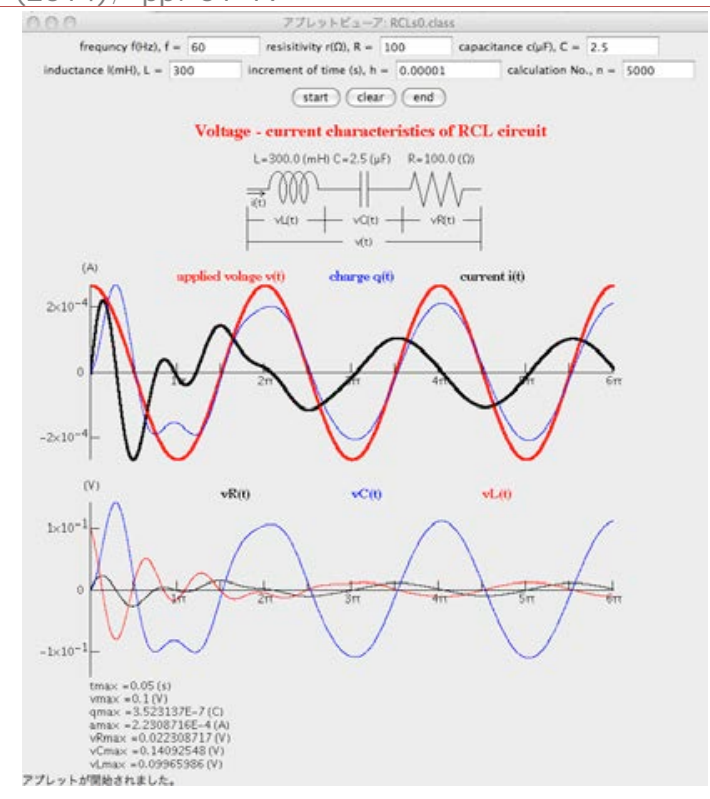

Figure 5: Updated AC characteristics of a series RCL circuit affected strongly by the capacitance after decreasing the value of $\mathrm{C}$ by a factor of 10 relative to that shown in Figure 4 . The phase of the current is less than that of the applied voltage by $\pi / 2$ and larger part of the applied voltage is applied to the capacitance

The typical DC characteristic, which corresponds to applying a rectangle voltage pulse, of the series RCL circuit in which the resistance, capacitance, and inductance are comparably effective are shown in Figure 6, using the same values in the text fields shown in Figure 4. By increasing the value of $R$ in the text field by a factor of 10 , relative to that shown in Figure 6 , the new characteristics affected strongly by the resistance, in which the shape of the current is similar to that of the applied voltage and the larger part of the voltage is applied to the resistance except in the transient regions, can be obtained immediately, as shown in Figure 7. By increasing the value of $L$ in the text field by a factor of 10 relative to that shown in Figure 6 , the new characteristics affected strongly by the inductance, in which the shape of the current is shown as a integration of the applied voltage and the larger part of the voltage is applied to the inductance, can be obtained immediately, as shown in Figure 8 . By decreasing the value of $\mathrm{C}$ by a factor of 10 and decreasing the value of $L$ by a factor of 100 , the new characteristics affected strongly by the capacitance, in which the shape of the current is shown as a differentiation of the applied voltage and the larger part of the voltage is applied to the capacitance, can be obtained immediately, as shown in Figure 9. 


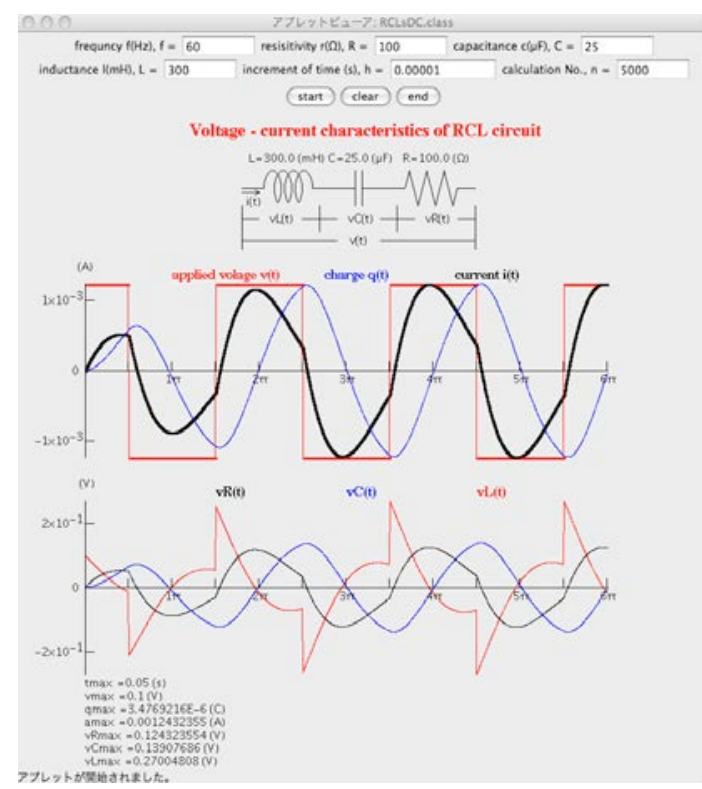

Figure 6: Typical DC characteristics of a series $\mathrm{RCL}$ circuit in which the $\mathrm{R}, \mathrm{C}$ and $\mathrm{L}$ components are comparably effective. The values used are same as those shown in Figure 4

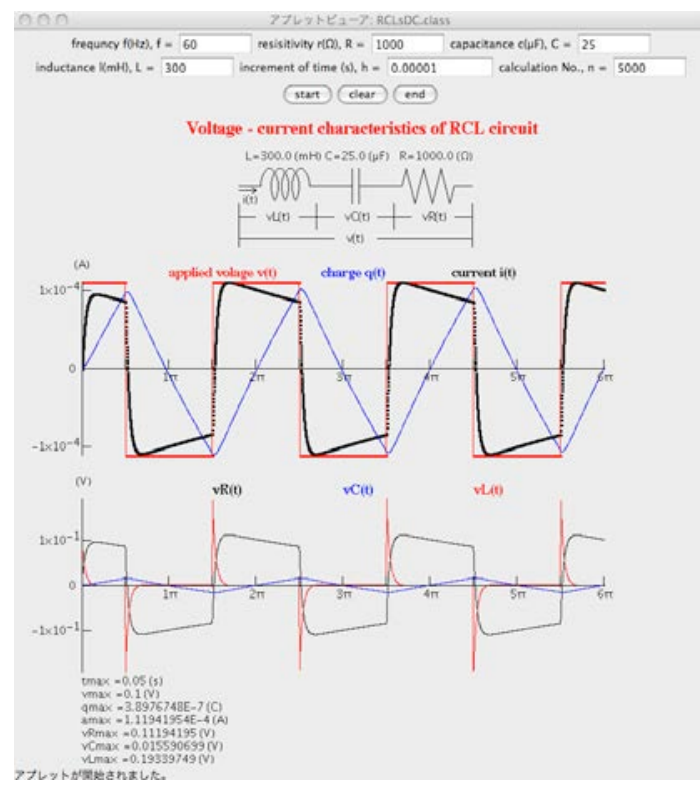

Figure 7: Updated DC characteristics of a series RCL circuit affected strongly by the resistance after increasing the value of $\mathbf{R}$ by a factor of 10 relative to that shown in Figure 6 . The shape of the current is similar to that of the applied voltage 


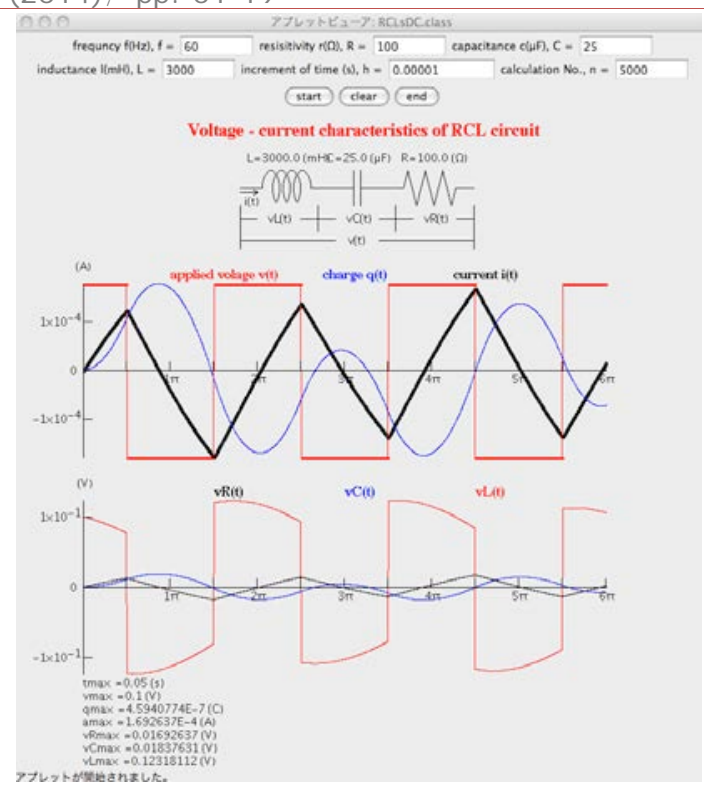

Figure 8: Updated DC characteristics of a series RCL circuit affected strongly by the inductance after increasing the value of $L$ by a factor of 10 relative to that shown in Figure 6 . The shape of the current is shown as an integration of the applied voltage

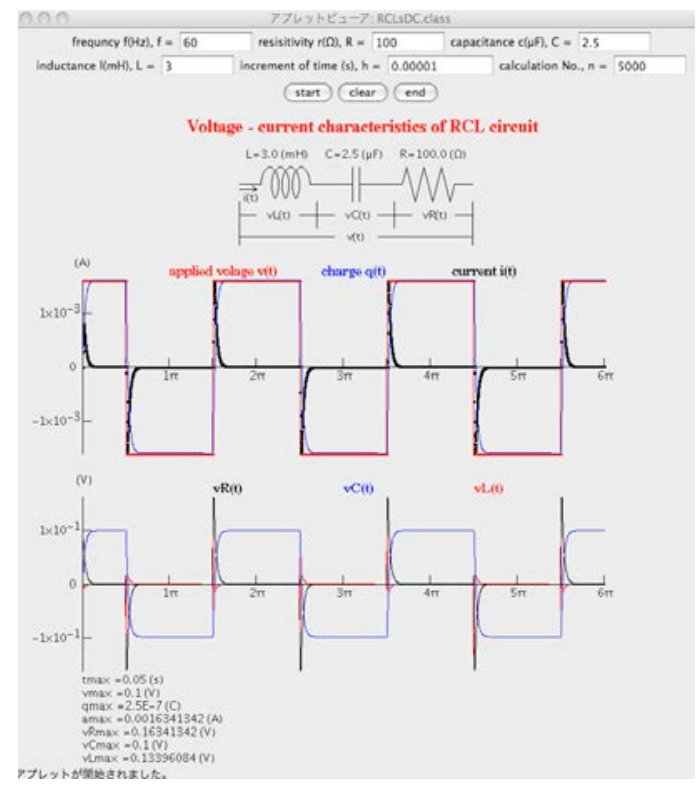

Figure 9: Updated DC characteristics of series RCL circuit affected strongly by the capacitance after decreasing the value of $C$ by a factor of 10 and decreasing the value of $L$ by a factor of 100 relative to the values shown in

Figure 6 . The shape of the current is shown as a differentiation of the applied voltage

\subsection{Electric Characteristics of Parallel RCL Circuit}

The typical AC characteristics of a parallel RCL circuit in which the resistance, capacitance, and inductance are comparably effective is shown in Figure 10. The used values in the text fields are same as the values used in Figure 4, except for the addition of $R_{S}$. The total current is dominated by the resistivity $R$, found by deducing the sum of the currents in the capacitance 
and the inductance, and the total current results in the same phase as the applied voltage. The phase of the charge is same as that of the current, that is, it is in phase with the capacitance voltage. The phase of the resistance current is same as that of the voltage. On the other hand, the phase of the capacitance current is smaller than that of the resistance current by $\pi / 2$, and that of the inductance current is larger than that of the resistance current by $\pi / 2$. The phase of the capacitance current is $\pi$ behind that of the inductance current. These basic electric characteristics of $R, C$, and $L$ can be easily shown visually on the display. By increasing the value of $C$ in the text field by a factor of 10 , the new characteristics are affected strongly by the capacitance because the capacitive impedance decreases by a factor of 10 in comparison with the other impedances. Thus, the phase of total current is less than that of the applied voltage by nearly $\pi / 2$, and the larger part of the total current is caused by the capacitance current. Theses characteristics can be obtained immediately, as shown in Figure 11 . In this case, the initial transient current results in very large values, as shown in Figure 11. By decreasing the value of $R$ in the text field by a factor of 10 relative to that in Figure 10, the new characteristics affected strongly by the resistance can be obtained immediately, and by decreasing that of $L$ by a factor of 10 , the new characteristics affected strongly by the inductance can also be obtained immediately. That is, by changing the values in the text fields and re-clicking the start button, the change in the characteristics of a parallel RCL circuit are recognized easily and accurately as an image showing the characteristics on the display.

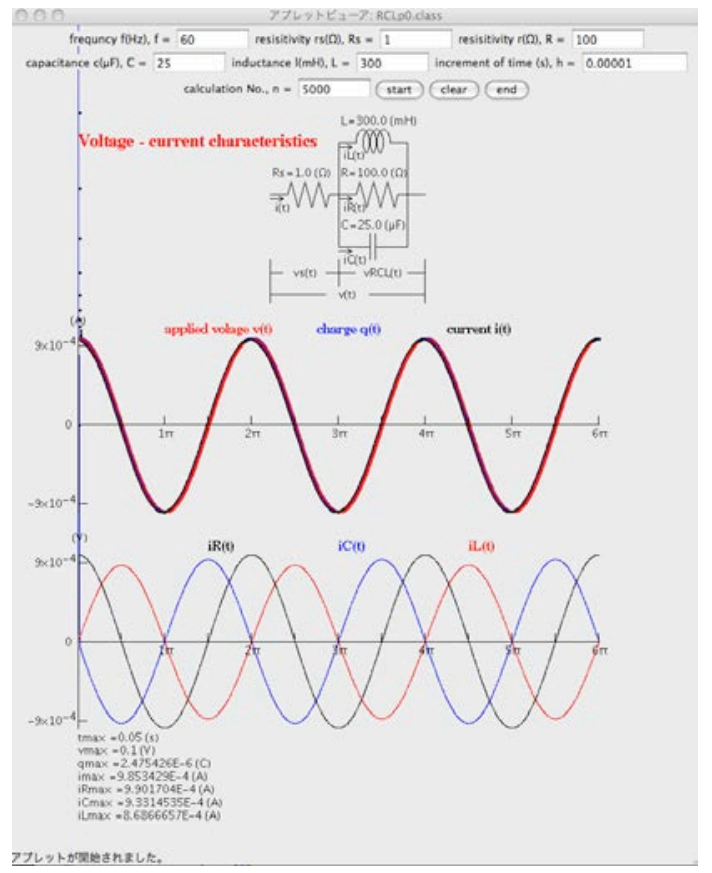

Figure 10: Typical AC characteristics of a parallel RCL circuit in which $R, C$ and $L$ components are comparably effective. The blues used are shown in the text fields, $f=60 \mathrm{~Hz}, R_{s}=1 \Omega, R=100 \Omega, C=25 \mu F$, and $L=300 \mathrm{mH}$. $v(t), i(t)$, and $q(t)$ are shown in the upper regions, and $i_{R}(t), i_{C}(t)$, and $i_{L}(t)$ are shown in the lower regions. The total current results in similar phase to the applied voltage, and the phase of the current in each component is different by $\pi / 2$ each other 


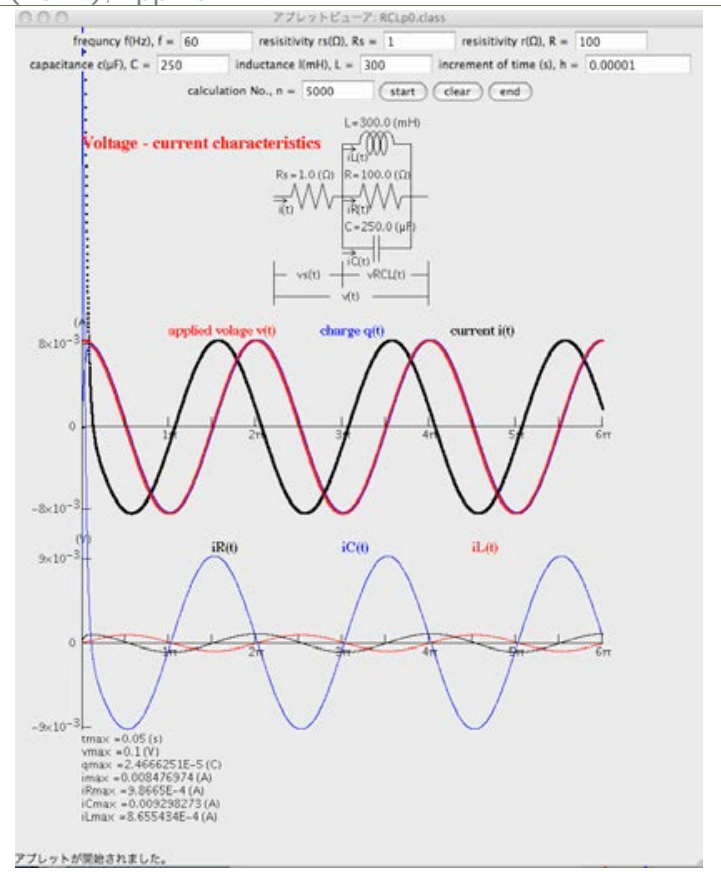

Figure 11: Updated AC characteristics of a parallel RCL circuit affected strongly by the capacitance after increasing the value of $\mathrm{C}$ by a factor of 10 relative to that shown in Figure 10. The phase of total current is less than that of the applied voltage by nearly $\pi / 2$, and the larger part of the total current is caused by the capacitance current

The typical DC characteristics, which corresponds to applying a rectangle voltage pulse, of a parallel RCL circuit, in which the resistance, capacitance, and inductance are comparably effective, is shown in Figure 12 using same values in the text fields as the AC characteristics shown in Figure 10. In the parallel circuit, the transient current of the capacitance is very large at the time when the rectangle voltage is changing. The maximum transient current is inversely proportional to $R_{S}$ in Figure 3 and is usually very large as shown in Figure 12 because of the small value of the $R_{s}$. The characteristics except in the transient regions are not obvious because the values are too small in contrast to the transient values. By decreasing the value of $\mathrm{R}$ in the text field by a factor of 10 relative to that shown in Figure 12, the new characteristics affected strongly by the resistance except in the transient regions, can be obtained immediately, as shown in Figure 13, in which the shape of the current is similar to that of the applied voltage and the larger part of the current is caused by the resistance current. By decreasing the value of $L$ in the text field by a factor of 10 relative to that shown in Figure 12, the new characteristics affected strongly by the inductance except in the transient regions, can be obtained immediately, as shown in Figure 14, in which the shape of the current is shown as an integration of the voltage and the larger part of the current is caused by the inductance current. By increasing the value of $\mathrm{C}$ by a factor of 10 relative to that shown in Figure 12, the new characteristics affected strongly by the capacitance, in which the shape of the current is shown as a differentiation of the voltage and the larger part of the current is caused by the capacitance current, can be obtained immediately, as shown in Figure 15. 


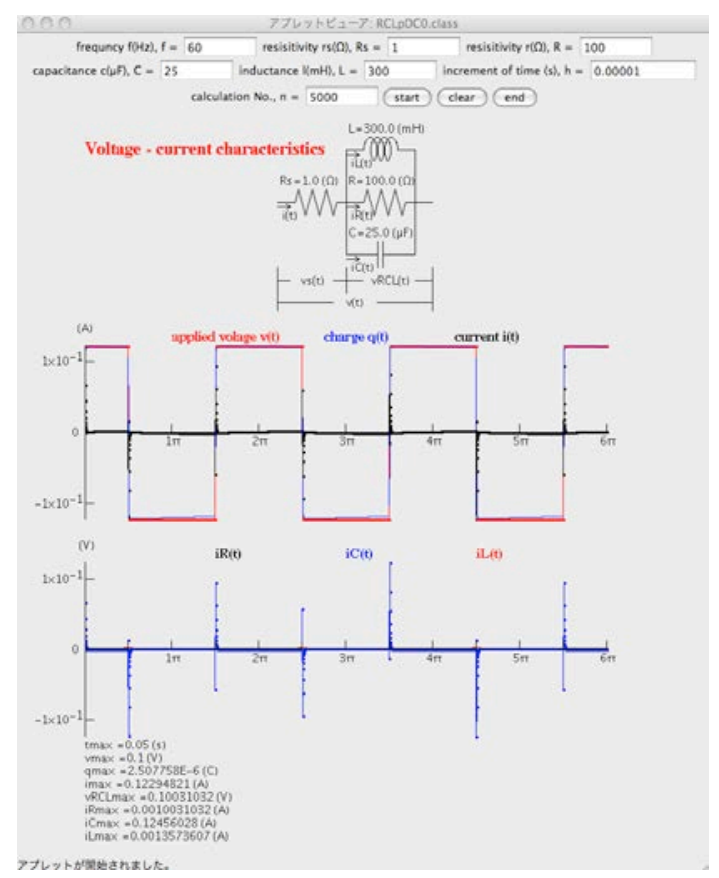

Figure 12: Typical DC characteristics of a parallel $\mathrm{RCL}$ circuit in which $\mathrm{R}, \mathrm{C}$ and $\mathrm{L}$ components are comparably effective. The values used are same as those shown in Figure 10. The transient current of the capacitance is very large and the characteristics, except in the transient regions, are not obvious because the values are too small in contrast to the transient values

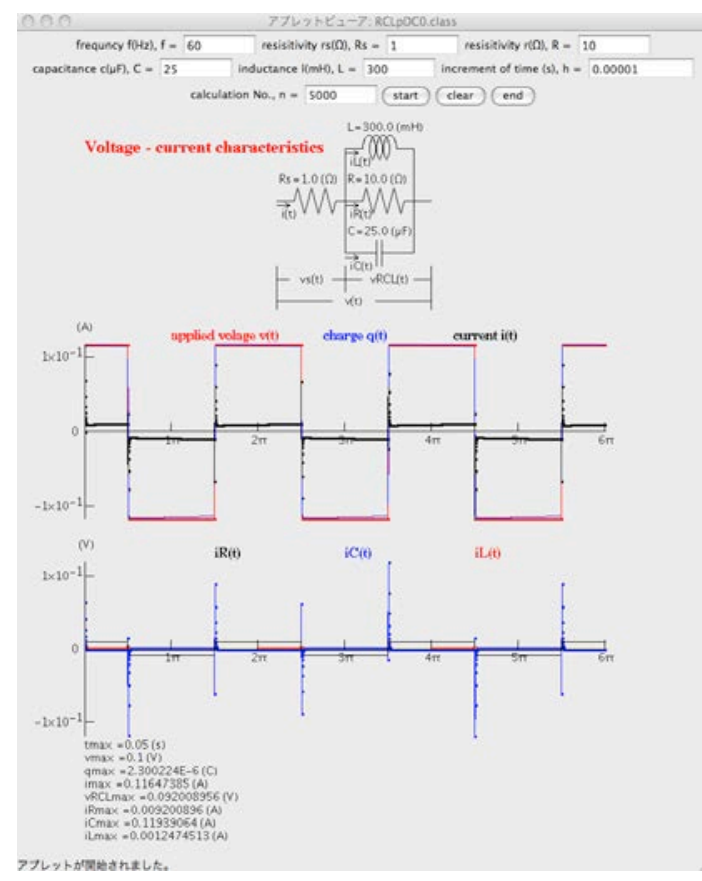

Figure 13: Updated DC characteristics of a parallel RCL circuit affected strongly by the resistance after decreasing the value of $\mathrm{R}$ by a factor of 10 relative to that shown in Figure 12. The shape of total current is similar to that of the voltage and the larger part of the current is caused by the resistance current, except in the transient regions 


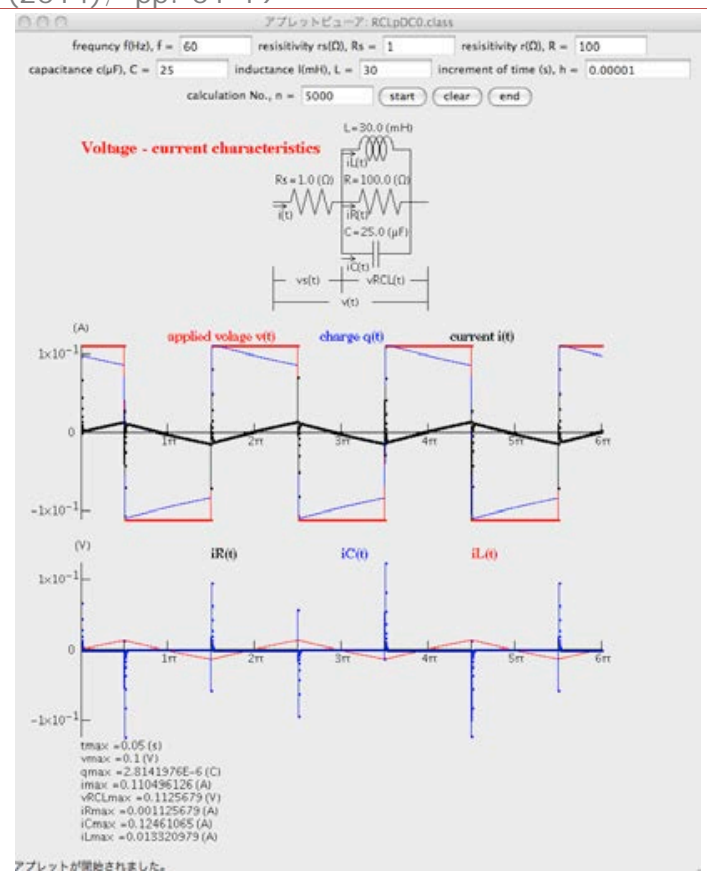

Figure 14: Updated DC characteristics of a parallel RCL circuit affected strongly by the inductance after decreasing the value of $L$ by a factor of $\mathbf{1 0}$ relative to that shown in Figure 12 . The shape of total current is shown as an integration of the voltage and the larger part of the current is caused by the inductance current, except in the transient regions

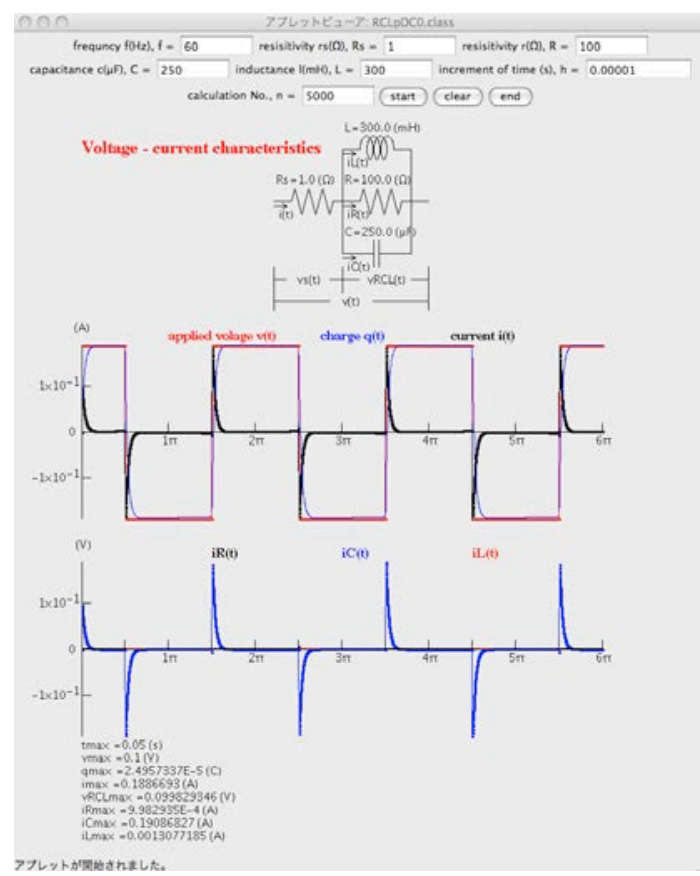

Figure 15: Updated DC characteristics of a parallel RCL circuit affected strongly by the capacitance after increasing the value of $\mathrm{C}$ by a factor of $\mathbf{1 0}$ relative to that shown in Figure 12. The shape of total current is shown as a differentiation of the voltage and the larger part of the current is caused by the capacitance current 


\section{DISCUSSION}

The accuracy of the calculations using this program depend on the value of the time increment, $h$. If we use a too large $h$, the calculation is not carried out accurately and the electric characteristics cannot be obtained, as shown in Figure 16. The accuracy of the calculation is increased by using smaller $h$ values. However, this leads to a larger number of calculations, thus a longer calculation time is needed to obtain the appropriate characteristics. It is better to choose a large $h$ value by performing tentative calculations with a relatively small number of calculations.

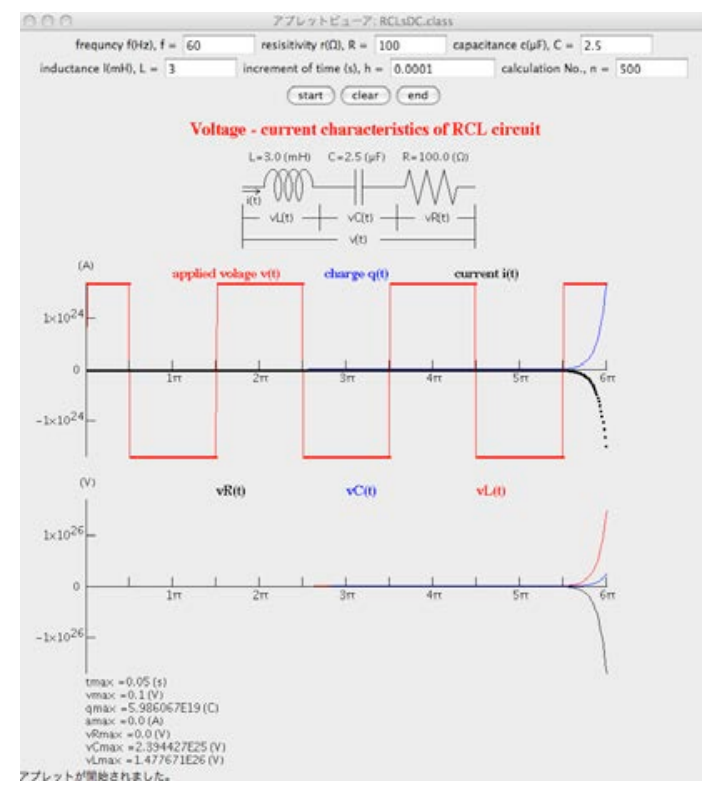

Figure 16: A sample of a non-accurate calculation using a large $h$ value. The values used for the calculation are same as those in Figure 9, except for $h=0.0001$ and $n=500$

The transient current of the capacitance is very large at the time when the changes in the voltage are large, as mentioned above. This results in the currents in the other components becoming relatively small such that their characteristics cannot be clearly presented, as shown in Figure 12. In this case, the characteristics can be clearly presented by selecting the maximum and minimum points on the display from the currents except in the transient current, as shown in Figure 17. 


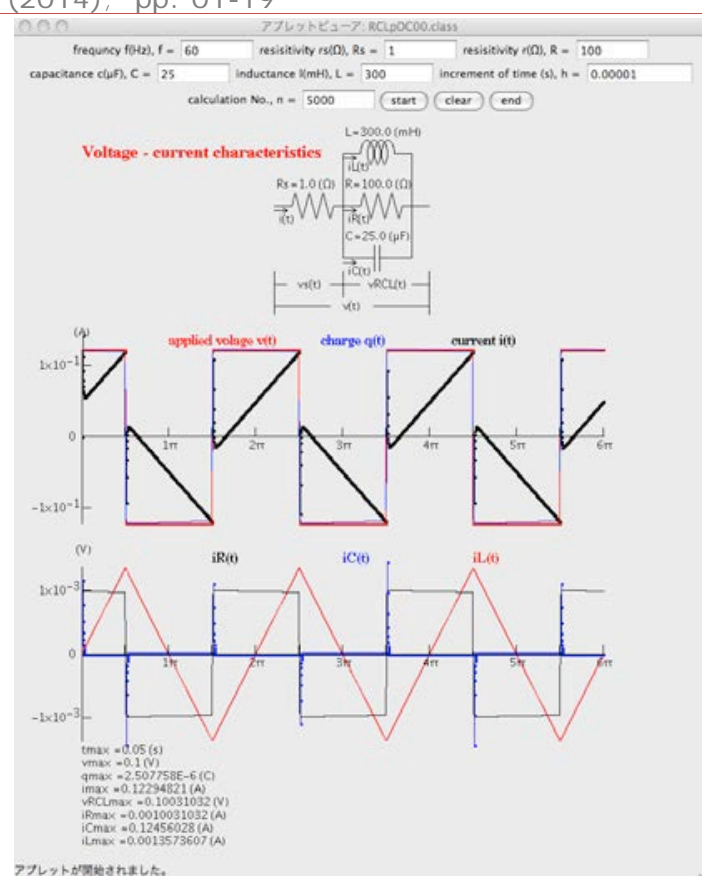

Figure 17: Selection of the maximum and minimum points on the display from the currents except in the transient current in the capacitance. The values used for the calculation are same as those in Figure 12

A typical oscillation of current in a series LC circuit applied a DC voltage is shown in Figure 18. The observed frequency of the oscillation, $318 \mathrm{~Hz}$, agrees with the theoretical value obtained using $1 /(2 \pi \sqrt{L C})$.

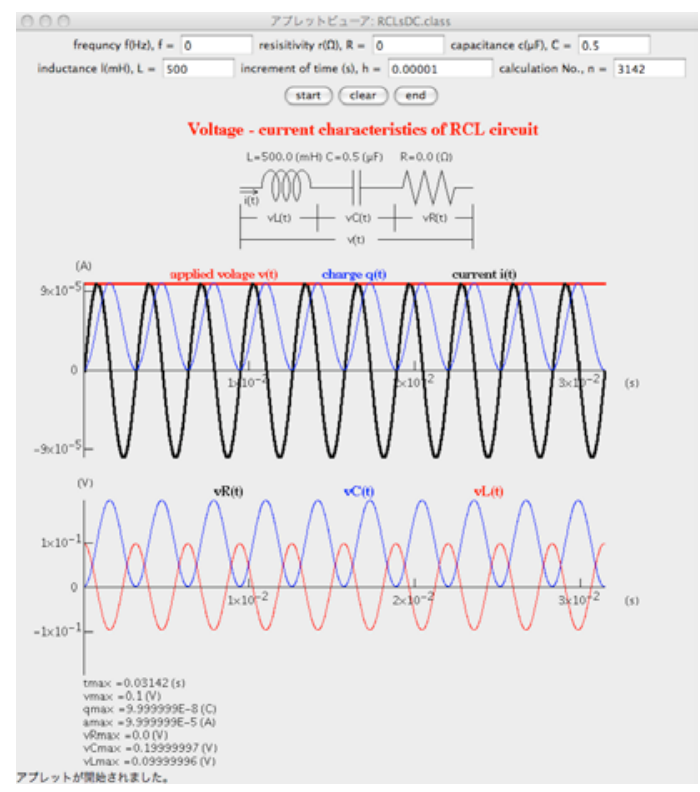

Figure 18: The typical oscillations of the current in a series LC circuit with a DC voltage applied

\section{CONCLUSION}

Java programs in a GUI environment have been developed to simulate the electric characteristics of a resistance, a capacitance, a inductance, and their circuits. The values of the 
selected parameters for the numerical calculation are set using the text fields on the display, and the calculation is initiated by clicking the start button after inputting these values. The calculated results are plotted immediately after the completion of the calculation as the figures of the voltage and current on the display. By changing the values in the text fields and reclicking the start button, the new results can be displayed immediately. The simulations of the characteristics depending on the values of each component in the electric circuit can be obtained easily and accurately as the changes of the voltage and current with time on the display. The time required to run a simulation is very short, less than several seconds using a personal computer. These Java programs are useful in education applications for image learning about the characteristics of electric circuits because of their ability to quickly provide accurate depictions of the fundamentals of electric circuits.

This Java program can be applied for rapidly and accurately image learning about the phenomena expressed by ordinary differential equations, because the Runge-Kutta method is used for the calculations. By using the Crank-Nicolson's implicit method and the Gauss-Seidel's iteration method, these Java simulations are also useful in education applications for rapidly and accurately image learning about complicated phenomena expressed by partial differential equations such as the diffusions of atoms [2] and the propagations of heat and wave.

\section{REFERENCES}

[1]. M. Morooka, S. Qian, and M. Morooka, Image Learnig of Charge Motion in Electric and Magnetic Fields by Java Programming, Transactions on Machine Learnig and Artificial Intelligence, 2014. 2(2): p.1- 19.

[2]. M. Morooka, Java Simulation of Au Diffusion in Si Affected by Vacancies and Self-Interstitials: Partial Differential Equations. Journal of Sofware Engineering and Applications, 2012. 5(10): p. 764-776. 